\title{
Asociación de la composición corporal total con la fuerza del tronco, el dolor y la discapacidad en pacientes con espondiloartrosis lumbar
}

\author{
The association of total body composition with trunk strength, pain and disability in \\ patients with lumbar osteoarthritis
}

\author{
Tania I. Nava-Bringas ${ }^{*}$, Laura López-Domínguez, Salvador I. Macías-Hernández, \\ Rolando Espinosa-Morales², Daniel D. Chávez-Arias ${ }^{3}$ y Roberto Coronado-Zarco ${ }^{4}$ \\ 'División de Rehabilitación Ortopédica; '2Departamento de Reumatología. Centro Colaborador de la Organización Panamericana de la Salud/ \\ Organización Mundial de la Salud para la Investigación y Rehabilitación Médica; ${ }^{3}$ Dirección Médica; ${ }^{4}$ Subdirección de Medicina de Rehabilitación. \\ Instituto Nacional de Rehabilitación Luis Guillermo Ibarra Ibarra, Ciudad de México, México
}

\section{Resumen}

Introducción: Las alteraciones en la composición corporal total podrían influir sobre la fuerza, el dolor y la discapacidad en pacientes con espondiloartrosis lumbar. Objetivo: Analizar la asociación de la composición corporal total con la fuerza muscular del tronco, el dolor y la discapacidad en pacientes con espondiloartrosis lumbar. Método: Estudio piloto en mayores de 50 años con dolor crónico de espalda baja y espondiloartrosis lumbar. Se excluyeron pacientes con diabetes mellitus, depresión, ansiedad, artropatías inflamatorias, fracturas vertebrales, escoliosis, cirugías de columna, cardiopatías, hipertensión arterial, radiculopatía o claudicación neurogénica. Se recolectaron datos sobre tiempo de evolución, composición corporal (masa grasa y muscular total), fuerza del tronco (isocinesia), dolor (escala numérica verbal) y discapacidad (Roland Morris). Análisis estadístico con $U$ de Mann-Whitney y correlaciones de Spearman. Resultados: 27 pacientes (18 mujeres y 9 hombres) con edad de $58.59 \pm 6.98$ años. La masa muscular total se asoció con el dolor (rho: $-0.63, p=0.001$ ) y con la fuerza del tronco (flexores rho: $-0.42, p=0.02$; extensores rho: $-0.50, p=0.007$ ), sin correlación con la discapacidad. No se encontró correlación de la masa grasa con ninguna de las variables. Conclusión: La disminución de la masa muscular se asocia con el dolor, pero no con la discapacidad, en pacientes con espondiloartrosis lumbar.

PALABRAS CLAVE: Composición corporal. Fuerza. Dolor. Discapacidad. Espondiloartrosis lumbar.

\begin{abstract}
Background: Variations in body composition among patients with lumbar osteoarthritis may influence pain and disability and muscle strength. Objective: To analyze the relationship between body composition with pain, disability and muscle strength, in patients with lumbar osteoarthritis. Methods: Pilot study in patients older than 50 years of age, with chronic low back pain and lumbar osteoarthritis, who agreed to participate through informed consent. We excluded patients with diabetes mellitus, depression, anxiety, inflammatory arthropathies, vertebral fractures, idiopathic scoliosis, spinal surgery, heart disease or hypertension, radiculopathy or neurogenic claudication. Data on evolution time, body composition (total body fat and muscle mass),
\end{abstract}

\author{
Correspondencia: \\ *Tania I. Nava-Bringas \\ Av. México-Xochimilco 289 \\ Col. Arenal de Guadalupe, Del. Tlalpan \\ C.P. 14389, Ciudad de México, Mexico \\ E-mail: tanianava@gmail.com
}

Fecha de recepción: 29-11-2017

Fecha de aceptación: 26-05-2018

DOI: $10.24875 / C I R U .18000006$
Cir Cir. 2018;86:388-391

Contents available at PubMed www.cirugiaycirujanos.com 
trunk strength, pain (numerical rating scale), and disability (Roland Morris questionnaire) were collected. Mann-Whitney U-test and Spearman correlations were performed. Results: 27 patients (18 women and 9 men) aged $58.59 \pm 6.98$ years. Negative correlations between muscle mass with pain (rho: $-0.63, p=0.001$ ) and strength (flexors rho: $-0.42, p=0.02$; extensors rho: $-0.50, p=0.007)$ were found, without correlation with disability. No correlations of fat mass with pain or disability were found. Conclusion: Decreased of muscle mass were associated with higher pain scores without influencing the disability in patients with lumbar osteoarthritis..

KEY WORDS: Body composition. Strength. Pain. Disability. Lumbar osteoarthritis.

\section{Introducción}

La espondiloartrosis hace referencia a los cambios degenerativos que suceden en la columna vertebral como consecuencia de la osteoartritis. Es una de las principales causas de dolor crónico de espalda baja y discapacidad en mayores de 50 años'.

La espondiloartrosis afecta primordialmente a las facetas articulares (articulaciones cigapofisiarias), aunque suele encontrarse asociada degeneración del disco intervertebral, y es uno de los principales factores de riesgo para la subsecuente degeneración de las facetas articulares ${ }^{2,3}$.

Hasta un $67 \%$ de los mayores de 50 años presentan espondiloartrosis lumbar en estudios de imagen, pero no todos experimentan dolor o limitación funcional, e incluso las manifestaciones clínicas varían de un sujeto a otro sin una relación directa con el grado de osteoartritis presente ${ }^{4,5}$.

Una posible explicación a estas variaciones clínicas es la presencia de alteraciones en la composición corporal total (CCT) de los individuos afectados de espondiloartrosis, primordialmente las relacionadas con cambios en los tejidos adiposo y muscular.

Existen estudios que han mostrado asociación de la morbimortalidad ocurrida en el envejecimiento con las alteraciones de la $\mathrm{CCT}^{6,7}$. De igual manera, algunos estudios realizados en patologías específicas, como la osteoartritis de rodillas, han sugerido que las alteraciones en la CCT pueden actuar como factores de riesgo independientes de la carga mecánica articular, modificando el dolor experimentado, la funcionalidad y el curso clínico de la enfermedad ${ }^{8,9}$.

Recientemente fue publicado un estudio que encontró asociación entre el grado de infiltración grasa en los músculos paraespinales y el grado de artrosis lumbar, pero en dicho estudio no se tomaron en cuenta variables clínicas como el dolor o el grado de discapacidad presentado por los pacientes con espondiloartrosis ${ }^{10}$.
Al momento no existe información disponible sobre la asociación entre las alteraciones en la CCT con el dolor y la discapacidad funcional de los pacientes con dolor crónico secundario al diagnóstico de espondiloartrosis lumbar, por lo cual el objetivo del presente estudio fue realizar un análisis preliminar de estos aspectos.

\section{Método}

Se realizó un estudio piloto, con muestreo no probabilístico por conveniencia en sujetos mayores de 50 años, de ambos sexos, con diagnóstico radiográfico de espondiloartrosis lumbar y presencia de dolor crónico lumbar (más de 3 meses de evolución), atendidos en el servicio de rehabilitación de columna del Instituto Nacional de Rehabilitación Luis Guillermo Ibarra Ibarra.

El protocolo de esta investigación fue previamente aprobado y registrado en el Comité de Investigación institucional, con número de registro 69/15. Todos los pacientes participaron de manera voluntaria, para lo cual se realizó la firma de un consentimiento informado.

Para el diagnóstico radiográfico se consideró la presencia de cuando menos dos segmentos funcionales de columna lumbar con hallazgos sugestivos de artrosis facetaria (reducción de espacios articulares, esclerosis, formación de osteofitos), pudiendo encontrarse también datos de degeneración discal (reducción de espacios intersomáticos y esclerosis de plataformas).

Se excluyeron pacientes con comorbilidad como diabetes mellitus, depresión, ansiedad, enfermedades reumáticas inflamatorias, antecedentes de fracturas vertebrales, escoliosis idiopática, cirugías previas de columna, cardiopatía isquémica o hipertensión arterial descontrolada, así como aquellos con datos clínicos de compromiso radicular agudo o estenosis lumbar (claudicación neurogénica).

Se recolectaron datos sobre la edad y el tiempo de evolución. El dolor fue evaluado mediante la 
escala numérica verbal, solicitando al paciente que eligiera el número que mejor representara el dolor experimentado en los últimos 7 días, considerando 0 como ausencia de dolor y 10 como el peor dolor posible. Para evaluar la discapacidad se aplicó el cuestionario de Roland-Morris, que es un instrumento de 24 ítems, autoaplicable y que refleja la limitación en las actividades de la vida diaria relacionadas con el dolor lumbar crónico. El puntaje total puede variar desde 0 (sin discapacidad) hasta 24 (máxima discapacidad).

Para evaluar la fuerza de los músculos del tronco se realizaron pruebas concéntricas mediante isocinesia en el equipo CON-TREX y el siguiente protocolo: previo calentamiento aeróbico submáximo consistente en caminata libre de 5 minutos, se situó al sujeto en bipedestación sobre la plataforma del equipo, verificando la correcta alineación sagital y coronal con respecto al eje rotacional del tronco (a nivel de L5); se colocaron sujeciones por encima y debajo de la patela, en las cinturas pélvica y escapular, y se realizó una calibración automática del equipo; se estableció un arco total de movimiento de $20^{\circ}$ de flexo-extensión del tronco para la realización de la prueba, y se hicieron dos pruebas a $40 \%$ de velocidad angular, cada una de tres repeticiones máximas. Al término de la prueba se extrajeron los datos sobre el pico de torque en Newtons por metro para estimar la fuerza de los grupos musculares flexores y extensores.

Para evaluar la CCT se utilizó impedancia bioeléctrica en un equipo Imbody $720^{\circledR}$, mediante el siguiente protocolo: previa eliminación de objetos metálicos del cuerpo, se colocó al paciente sin calzado sobre el equipo y sujetando con ambas manos los sensores, tomando una lectura por 4 minutos, con lo cual se extrajeron para análisis los valores del índice de masa corporal (IMC), la masa grasa total en kilos, el porcentaje de grasa corporal y la masa muscular esquelética en kilos. El equipo determina, de acuerdo con el IMC, el valor normal establecido para cada sujeto sobre la masa grasa total y la masa muscular esquelética, arrojando los resultados para comparar si se encuentra en parámetros normales o alterados.

El análisis de la información se realizó con el paquete estadístico SPSS versión 21. Se realizó estadística descriptiva para resumir los datos; se eligieron pruebas no paramétricas ( $\mathrm{U}$ de Mann Whitney) para contrastación de datos y correlaciones de Spearman para asociación de variables.
Tabla 1. Fuerza muscular (picos de torque en Newtons) de los músculos flexores y extensores del tronco

\begin{tabular}{lccc}
\hline & Sexo & Media & p \\
\hline Flexores del tronco & Femenino & $115.0 \pm 32.8 \mathrm{~N}$ & 0.23 \\
& Masculino & $98.95 \pm 30.42$ & \\
& & $\mathrm{~N}$ & \\
& & $\mathrm{~N}$ & \\
Extensores del & Femenino & $139.54 \pm 37.57$ & 0.46 \\
tronco & & $\mathrm{N}$ & \\
& Masculino & $126.65 \pm 52.27$ & \\
& & $\mathrm{~N}$ & \\
\hline
\end{tabular}

Tabla 2. Resultados del análisis de la composición corporal total

\begin{tabular}{lllll}
\hline & Sexo* $^{*}$ & Media & DE & $\mathbf{p}^{\dagger}$ \\
\hline Índice de masa corporal & Femenino & 27.53 & 4.0 & 0.64 \\
& Masculino & 31.0 & 4.98 & \\
Masa muscular & Femenino & 25.27 & 4.97 & 0.36 \\
esquelética $(\mathrm{kg})$ & & & & \\
& Masculino & 23.52 & 3.84 & \\
Masa grasa corporal (kg) & Femenino & 29.04 & 8.01 & 0.88 \\
& Masculino & 29.03 & 10.2 & \\
Porcentaje de grasa corporal & Femenino & 38.13 & 6.72 & 0.41 \\
& Masculino & 40.53 & 7.66 & \\
\hline
\end{tabular}

*18 mujeres y 9 hombres.

+U de Mann Whitney para comparar medias entre sexos.

DE: desviación estándar.

\section{Resultados}

Se incluyeron 27 sujetos (18 mujeres y 9 hombres) con una edad promedio de $58.59 \pm 6.98$ años. El tiempo de evolución de los síntomas de dolor era de $1.72 \pm 1.59$ años. No se encontraron diferencias significativas en los promedios de edad y de tiempo de evolución entre sexos.

La intensidad del dolor medida por la escala numérica fue de $6.56 \pm 1.73$ puntos de promedio, y la discapacidad evaluada con la escala de Roland Morris obtuvo un promedio de $9.7 \pm 3.59$ puntos para el grupo, sin diferencias entre sexos $(p \geq 0.05)$. Los resultados de la fuerza (isocinesia) de la musculatura del tronco y de los porcentajes de tejido muscular y adiposo (CCT por impedanciometría) se muestran en las tablas 1 y 2 , respectivamente.

En el análisis de correlación se buscó la asociación de los puntajes de dolor con la fuerza de los músculos flexores (rho: $-0.42, p=0.02$ ), la fuerza de los músculos extensores (rho: $-0.50, p=0.007$ ), la masa muscular en kilos (rho: $-0.63, p=0.001$ ), la masa grasa en 
kilos (rho: $-0.239, p=0.230$ ), el porcentaje de grasa corporal total (rho: $0.086, p=0.67$ ), el IMC (rho: 0.027 , $p=0.89$ ) y la edad (rho: $0.085, p=0.674$ ).

También se buscó la asociación de los puntajes obtenidos del cuestionario de Roland-Morris para funcionalidad con la fuerza de los músculos flexores (rho: $-0.115, p=0.567$ ), la fuerza de los músculos extensores (rho: $-0.245, p=0.218$ ), la masa muscular en kilos (rho: $-0.031, p=0.877$ ), la masa grasa en kilos (rho: $0.022, p=0.915$ ), el porcentaje de grasa corporal total (rho: $-0.030, p=0.883$ ), el IMC (rho: -0.112 , $p=0.577$ ) y la edad (rho: $0.238, p=0.232$ ).

\section{Discusión}

La información obtenida en este estudio piloto confirma la importancia del tejido muscular en los pacientes con dolor crónico y espondiloartrosis lumbar; estudios previos realizados mediante resonancia magnética en sujetos con dolor crónico de espalda baja han relacionado la atrofia muscular con la presencia de dolor crónico ${ }^{7,11}$.

En cuanto al tejido adiposo y sus implicaciones en la osteoartritis, se ha observado una tendencia al incremento en publicaciones que mencionan la influencia del tejido adiposo sobre la evolución natural de la osteoartritis, siendo incluso un factor de riesgo independiente de la carga mecánica articular, con principal información en osteoartritis de rodillas y manos; al momento contamos con poca información disponible sobre columna vertebral ${ }^{8,9,12}$.

Solo Urquhart, et al. ${ }^{13}$ realizaron un estudio de CCT mediante impedanciometría, en 135 sujetos reclutados de clínicas de obesidad (edades entre 25 y 62 años). En su estudio se aplicaron cuestionarios sobre dolor lumbar y discapacidad en los últimos 6 meses, y lo compararon con voluntarios sanos, encontrando una asociación del dolor y de la discapacidad con el incremento en el tejido adiposo; sin embargo, no se realizaron estudios de imagen de columna ni se estableció un diagnóstico específico.

En contraste con lo anterior, en el presente estudio no demostramos asociación del porcentaje de tejido adiposo y de la masa grasa total con las variables clínicas recolectadas (dolor y discapacidad), a pesar de que la gran mayoría de los sujetos del estudio presentaron valores superiores al esperado.
Estos hallazgos deben ser tomados con reserva. Nuestra principal limitante es la muestra reducida de pacientes y el tiempo de evolución inferior a 2 años, lo cual pudiera no representar todas las fases de la evolución natural de la espondiloartrosis. Por tal motivo, consideramos relevante ampliar el número de individuos y considerar las implicaciones del tejido adiposo que han sido reportadas en otros estudios en etapas avanzadas de la osteoartritis.

\section{Conclusión}

La disminución de la masa muscular se asocia con el dolor, pero no con la discapacidad, en pacientes con espondiloartrosis lumbar. No se encontró asociación entre las alteraciones en el tejido adiposo y el dolor o la discapacidad en pacientes con espondiloartrosis lumbar.

\section{Conflicto de intereses}

Los autores declaran no tener ningún conflicto de intereses para la realización del presente trabajo.

\section{Bibliografía}

1. Hunter DJ, Felson DT. Osteoarthritis. BMJ. 2006;332:639-42.

2. Gellhorn AC, Katz JN, Suri P. Osteoarthritis of the spine: the facet joints. Nat Rev Rheumatol. 2013;9:216-24.

3. Suri P, Miyakoshi A, Hunter DJ, Jarvik JG, Rainville J, Guermazi A, et al. Does lumbar spinal degeneration begin with the anterior structures? A study of the observed epidemiology in a community-based population. BMC Musculoskelet Disord. 2011;12:202.

4. Goode AP, Carey T, Jordan JM. Low back pain and lumbar spine osteoarthritis: how are they related? Curr Rheumatol Rep. 2013;15:305.

5. Hochberg MC, Altman RD, Brandt K, Clark B, Dieppe P, Griffin M, et al. Guidelines for the medical management of osteoarthritis. Arthritis Rheum. 1995;38:1541-6.

6. Francis P, Lyons M, Piasecki M, Mc Phee J, Hind K, Jakeman P. Measurement of muscle health in aging. Biogerontology. 2017;18:901-11.

7. Kalichman L, Carmeli E, Been E. The association between imaging parameters of the paraspinal muscles, spinal degeneration, and low back pain. Biomed Res Int. 2017;2017:2562957.

8. Zhuo Q, Yang W, Chen J, Wang Y. Metabolic syndrome meets osteoarthritis. Nat Rev Rheumatol. 2012;8:729-37.

9. Van der Esch M, Knoop J, van der Leeden M, Roorda LD, Lems WF $\mathrm{Knol} \mathrm{DL}$, et al. Clinical phenotypes in patients with knee osteoarthritis: a study in the Amsterdam osteoarthritis cohort. Osteoarthr Cartil. 2015;23:544-9.

10. Yu B, Jiang K, Li X, Zhang J, Liu Z. Correlation of the features of the lumbar multifidus muscle with facet joint osteoarthritis. Orthopedics. 2017;40:e793-e800.

11. Danneels LA, Vanderstraeten GG, Cambier DC, Witvrouw EE, De Cuyper HJ. CT imaging of trunk muscles in chronic low back pain patients and healthy control subjects. Eur Spine J. 2000;9:266-72.

12. Zhang $P$, Zhong ZH, Yu HT, Liu B. Significance of increased leptin expression in osteoarthritis patients. PLoS One. 2015;10:e0123224.

13. Urquhart DM, Berry P, Wluka AE, Strauss BJ, Wang Y, Proietto J, et al. Increased fat mass is associated with high levels of low back pain intensity and disability. Spine (Phila Pa 1976). 2011;36:1320-5. 